\title{
AN ELEMENTARY PROOF OF A THEOREM OF SCHAEFER, WOLFF AND ARENDT
}

\author{
C. B. HUIJSMANS
}

(Communicated by John B. Conway)

\begin{abstract}
An elementary proof of the following result, due to Schaefer, Wolff, and Arendt is given: if $T$ is a lattice homomorphism on a Banach lattice $E$ with spectrum $\sigma(T)=\{1\}$, then $T=I$, the identity mapping on $E$.
\end{abstract}

\section{INTRODUCTION}

It is shown by Schaefer, Wolff, and Arendt in [5] that a lattice homomorphism $T$ on a Banach lattice $E$ with spectrum $\sigma(T)=\{1\}$ is necessarily equal to the identity mapping $I$ on $E$. By considering the adjoint mapping of $T$ instead, one may assume without loss of generality that $E$ is Dedekind complete. Their proof is however far from simple and uses representation theory heavily.

Let $\mathscr{L}(E)$ denote the Banach space of all norm bounded linear operators on $E$ and $\mathscr{L}_{b}(E)(\subset \mathscr{L}(E))$ the Dedekind complete Banach lattice of all order bounded (= regular) linear operators on $E$, equipped with the regular norm. Recently, it was proved by Voigt [6] that the band projection $\mathscr{D}$ of $\mathscr{L}_{b}(E)$ onto the center $Z(E)$ of $E$ is a contraction with respect to the operator norm. It is the purpose of the present note to show that this result, combined with some observations from [2], can be used to give an elementary and representation-free proof of the above mentioned theorem of Schaefer, Wolff, and Arendt.

For the basic theory of vector lattices (Riesz spaces) and Banach lattices and for unexplained terminology and results we refer to [1,3,4, and 7].

\section{SOME PREREQUISITES}

Let $E$ be a Banach latticè. As is well known, the center

$$
Z(E)=\{\pi \in \mathscr{L}(E): \pm \pi \leq \lambda I \text { for some } \lambda>0\}
$$

is an Archimedean $f$-algebra with unit element $I$ (see e.g. [7, Theorem 140.9 and Corollary 144.3]). Moreover, if $\pi \in Z(E)$, then

$$
\|\pi\|=\||\pi|\|=\inf \{\lambda>0:|\pi| \leq \lambda I\},
$$

Received by the editors May 6, 1988.

1980 Mathematics Subject Classification (1985 Revision). Primary 46B30, 47B55; Secondary 06F25, 47A10, 47D05. 
where $\|\pi\|$ denotes the operator norm of $\pi$ [7, Corollary 144.3]. It follows easily that $\|\pi\|=r(\pi)$, the spectral radius of $\pi$. Indeed, $\pi^{2}=|\pi|^{2} \leq \lambda I$ is equivalent to $\| \pi \mid \leq \sqrt{\lambda} \cdot I$, as $Z(E)$ is semiprime [7, Theorems 142.3 and 142.5]. The equality $\left\|\pi^{2}\right\|=\|\pi\|^{2}$ is immediate and hence

$$
\|\pi\|=r(\pi)=\lim _{n \rightarrow \infty}\left\|\pi^{n}\right\|^{1 / n} \text {. }
$$

From now on, $E$ is Dedekind complete Banach lattice, $E \neq\{0\}$. Let $\mathscr{D}$ denote the band projection of $\mathscr{L}_{b}(E)$ onto $Z(E)$ ( $\mathscr{D}$ for diagonal part). Voigt shows in [6, Theorem 1.4] that $\|\mathscr{D}(S)\| \leq\|S\|$ for all $S \in \mathscr{L}_{b}(E)$, in other words, $\mathscr{D}$ is a contraction with respect to the operator norm. Apart from his proof of Lemma 1.2, all the proofs in this paper are free of representation. The next alternative proof of Lemma 1.2 of [6] is representation-free.

Lemma 1. If $\pi \in Z(E)$ and $\varepsilon>0$, then there exists a band $B \subset E, B \neq\{0\}$ such that

$$
\|\pi f\| \geq(\|\pi\|-\varepsilon)\|f\|
$$

for all $f \in B$.

Proof. On account of the equalities $\|\pi\|=\||\pi|\|$ and $|\pi f|=\| \pi|f|$ for all $f \in E$ we may assume without loss of generality that $\pi>0$. If $\varepsilon \geq\|\pi\|$, then $B=E$ fulfills the requirements, so we may restrict ourselves to the case $0<\varepsilon<\|\pi\|$. Put $\mu=\|\pi\|-\varepsilon$. It follows from

$$
\|\pi\|=\inf \{\lambda>0: 0<\pi \leq \lambda I\}
$$

and $0<\mu<\|\pi\|$ that $(\pi-\mu I)^{+}>0$, so there exists $0<v \in E$ such that $u=(\pi-\mu I)^{+} v>0$. Take $B=N\left((\pi-\mu I)^{-}\right)$, the null space of $(\pi-\mu I)^{-}$. Then $B$ is a band in $E$ and it follows from

$$
(\pi-\mu I)^{-}(\pi-\mu I)^{+}=0
$$

that $u \in B$, so $B \neq\{0\}$. If $0 \leq w \in B$, then

$$
(\pi-\mu I)^{-} w=(\pi w-\mu w)^{-}=0
$$

and thus $\pi w \geq \mu w=(\|\pi\|-\varepsilon) w$. If $f \in B$ is arbitrary, then $\pi|f| \geq(\|\pi\|-\varepsilon)|f|$ yields

$$
\|\pi f\|=\|\pi|f|\| \geq(\|\pi\|-\varepsilon)\||f|\|=(\|\pi\|-\varepsilon)\|f\|,
$$

which is the desired result.

We have therefore accomplished a representation-free proof of Voigt's result.

\section{The MAIN Result}

Let $T: E \rightarrow E$ be a lattice homomorphism on the Dedekind complete Banach lattice $E$ with $\sigma(T)=\{1\}$. Write $T=I+N$ with $\sigma(N)=\{0\}$ (i.e., $N$ is quasinilpotent). It follows from $T \geq 0$ and $N=T-I$ that $N \in \mathscr{L}_{b}(E)$. In the next proposition we list some results from [2] (Corollary 6, Proposition 7, and Proposition 10, respectively). 


\section{Proposition 2.}

(i) $N^{+} \in Z(E)^{d}, N^{-} \in Z(E)$ (even $\left.0 \leq N^{-} \leq I\right)$.

(ii) $N^{+}=N^{-} N^{+}=N^{+} N^{-}, N^{-}=\left(N^{-}\right)^{2}$.

(iii) $\left(N^{+}\right)^{k} \in Z(E)^{d}(k=1,2, \ldots)$.

We have now gathered all the tools for the elementary proof of [5, Corollary 2.2].

Theorem 3 (Schaefer-Wolff-Arendt). Let $E$ be a Banach lattice and $T: E \rightarrow E$ a lattice homomorphism for which $\sigma(T)=\{1\}$. Then $T=I$.

Proof. As observed before, we may assume that $E$ is Dedekind complete. Writing $T=I+N$ with $r(N)=0$, we have to show that $N=0$.

It follows from Proposition 2 (ii) that

$$
N^{k}=\left(N^{+}-N^{-}\right)^{k}=\sum_{i=0}^{k-1}\left(\begin{array}{l}
k \\
i
\end{array}\right)\left(N^{+}\right)^{k-i}\left(-N^{-}\right)^{i} \pm\left(N^{-}\right)^{k}
$$

$(k=1,2, \ldots)$. Consequently,

$$
\mathscr{D}\left(N^{k}\right)= \pm\left(N^{-}\right)^{k}= \pm N^{-}
$$

as

$$
\sum_{i=0}^{k-1}\left(\begin{array}{l}
k \\
i
\end{array}\right)\left(N^{+}\right)^{k-i}\left(-N^{-}\right)^{i} \in Z(E)^{d}
$$

by Proposition 2 (iii). Hence, by Voigt's result,

$$
\left\|\left(N^{-}\right)^{k}\right\|=\left\|\mathscr{D}\left(N^{k}\right)\right\| \leq\left\|N^{k}\right\|
$$

$(k=1,2, \ldots)$ and hence

$$
\left\|\left(N^{-}\right)^{k}\right\|^{1 / k} \leq\left\|N^{k}\right\|^{1 / k}
$$

$(k=1,2, \ldots)$. Taking limits, we find

$$
\left\|N^{-}\right\|=r\left(N^{-}\right) \leq r(N)=0 .
$$

Hence, $N^{-}=0$, so $N^{+}=N^{-} N^{+}=0$ as well. It follows that $N=0$ and the proof is complete.

\section{REFERENCES}

1. C. D. Aliprantis and O. Burkinshaw, Positive operators, Academic Press, Orlando, 1985.

2. C. B. Huijsmans, Elements with unit spectrum in a Banach lattice algebra, Indag. Math. $\mathbf{5 0}$ (1988), 43-51.

3. W. A. J. Luxemburg and A. C. Zaanen, Riesz spaces I, North-Holland, Amsterdam, 1971.

4. H. H. Schaefer, Banach lattices and positive operators, Springer, Berlin, 1974. 
5. H. H. Schaefer, M. Wolff, and W. Arendt, On lattice isomorphisms with positive real spectrum and groups of positive operators, Math. Z. 164 (1978), 115-123.

6. J. Voigt, The projection onto the center of operators in a Banach lattice, Math. Z. 199 (1988), 115-117.

7. A. C. Zaanen, Riesz spaces II, North-Holland, Amsterdam, 1983.

Department of Mathematics, Leiden State University, P.O. Box 9512, 2300 RA Leiden, THE NETHERLANDS 